\title{
VERSIÓN ACADIA DE LA INSCRIPCIÓN REAL A DE SAMSUILUNA, REY DE BABILONIA
}

\author{
Juan Antonio Pino Cano \\ (Universidad Complutense de Madrid)
}

\begin{abstract}
La presente contribución al volumen que honra al Prof. Federico Lara, quiere agradecer su esfuerzo en dar a conocer el antiguo Oriente en la Universidad española. Gratitud por sus clases y consejos que me han sido de gran ayuda y a nuestra larga amistad.
\end{abstract}

\section{RESUMEN}

Los reyes de la Primera Dinastía de Babilonia registran en los $<<$ Nombres de año $>>$ la destrucción, restauracióny construcción de murallas. Samsuiluna (1749-1712) incide en estapolítica arquitectónica de carácter defensivo. Asi lo pone de manifiesto la inscripción Real A de Samsuiluna al recoger la restauración de seis murallas que habian sido construidas por uno de sus predecesores, Sümû-1a-I1.

\section{PALABRAS CLAVE}

Samsuiluna, Sūmû-1a-I1,Hammurapi, Muralla, Primera Dinastía de Babilonia.

\section{ABSTRACT}

The kings of the First Dynasty of Babylon recorded in the $<<$ Names of the year $>>$ the demolition, restoration and construction of walls. Samsuiluna (1749-1712) insists on this architectural policy of a defensive nature. This is evidenced by the inscription Real A of Samsuiluna when he recalled the restoration of six walls that had been built by one of his predecessors, Sümû-la-Il.

\section{KEYWORDS}

Samsuiluna, Sūmû-1a-I1, Hammurapi, Wall, First Dynasty of Babylon.

\section{Precedentes.}

A la muerte de Hammurapi en el año $1750^{1}$, el legado que había dejado a su hijo Samsuiluna no se veía amenazado por ninguna parte, pero en poco menos de diez años las tensiones tanto externas (rebeliones) como internas (políticas y económicas) iban a desencadenar una desestabilización en una gran parte del imperio.

Hammurapi basó sus logros sobre su poderosa personalidad y un sistema administrativo que de manera progresiva iba disminuyendo los poderes locales para engrandecer los del rey. El reino alcanzado por Hammurapi se logró en un corto periodo de tiempo y englobaba a una amplia zona geográfica donde se ubicaban múltiples estados, todos ellos con una gran personalidad y prestigio desde antaño, entre ellos se encontraban: Larsa, Mari, Ešnunna y Aššur.

Tanto su hijo Samsuiluna como sus sucesores trataron de aplastar todas estas revueltas pero no lo consiguieron de una manera definitiva $\mathrm{y}$, poco a poco, el desmembramiento del imperio se iba consolidando. Esta situación desde el punto de vista económico produjo pérdidas de renta en las tierras que pasaban al enemigo. En definitiva, a los sucesores de Hammurapi no les quedó más iniciativa que intensificar tanto los impuestos en aquellas tierras que todavía conservaban como la producción agrícola ${ }^{2}$.

\footnotetext{
${ }^{1}$ Todas las fechas que se recogen en este artículo mientras que no se indique lo contrario son anteriores a la era cristiana.

${ }^{2}$ Para una visión de conjunto acerca de las pérdidas de renta de la tierra e impuestos de índole diversa, vid. Yofee 1977: 143-151.; Renger 1979: 252; Bottéro 1961: 113-164; Gibson 1974: 7-19.
} 


\section{Reinado de Samsuiluna}

Samsuiluna $^{3}$ (1749-1712) séptimo rey de la Primera Dinastía de Babilonia (Dinastía amorrita), hijo de Hammurapi poseyó el valor y constancia de su padre al hacer frente a las continuas rebeliones que surgieron, aunque no así su talla, en cuanto a astucia y administrador del Estado. La mayor parte de su reinado se centró en las expediciones militares que tuvo que realizar contra diversos pueblos provenientes de distintas zonas geográficas. También fue uno de los reyes de esta dinastía que más contribuyó a la realización de trabajos arquitectónicos de carácter defensivo, concretamente, en la restauración de antiguas murallas. Ello vino derivado de la compleja situación social y económica de gobernar un amplio imperio, sostenido por las armas. Durante el octavo año de reinado de Samsuiluna $(1742)^{4}$, Babilonia conoció la presión de los casitas ( $k a$ ššû), pueblo proveniente del este. Descendieron de las montañas de los Zagros empujados por los movimientos de pueblos indoeuropeos. Samsuiluna tuvo que hacerles frente y su atención puesta en este conflicto fue aprovechada por algunas ciudades del sur mesopotámico (Uruk, Ur y Larsa) para sublevarse, unidas a otras más septentrionales (Išin y Ešnunna). Las fuentes babilonias citan a un tal Rîm-Sîn, nombre que aludía al último y prestigioso soberano de Larsa, quien se apoderó de dicha ciudad. Con este logro RîmSîn alcanzaba anexionarse todo el sur mesopotámico y, al mismo tiempo, fomentar una insurrección en Iamutbal, cuna de sus antepasados. El sometimiento de Rîm-Sîn se logró cinco años después (1736) en la ciudad de Kiš. Su aliado, el príncipe de Ešnunna fue capturado y trasladado a Babilonia en una jaula donde fue estrangulado. Las fuentes añaden que durante el transcurso de esta expedición militar, Samsuiluna derribó las murallas de Ur y saqueó e incendió sus templos y gran parte de la ciudad. Igual suerte corrió la ciudad de Uruk lo que daría pie a los elamitas a entrar en el conflicto. Su rey Kutir-Nahhunte I (1730-1700) en una rápida incursión en la zona penetró en Uruk y se llevó a Susa, entre otros tesoros, la estatua de la diosa Inanna que casi mil años más tarde, el rey asirio Asurbanipal debió recuperar y trasladar a Nínive. Tal hecho militar permitió al rey Kutir-Naḩhunte conquistar y saquear más de 30 ciudades, incluso llegó a atacar la propia ciudad de Babilonia para vengar la derrota que infligió al país de Elam el rey Hammurapi.

Durante el año 1735 las fuentes hablan de la irrupción de un nuevo personaje, Iluma-ilu (1720-1695), que pretendía ser descendiente de Damiq-ilišu (1816-1794), último soberano de la ciudad de Isin de época paleo-babilónica. Agitó una revuelta en el país de Sumer, se apoderó de la ciudad de Nippur y consiguió repeler dos ataques de Samsuiluna. Finalmente, Iluma-ilu fundó una dinastía autóctona conocida como "Dinastía del Mar", constituida por residuos de sumerios y acadios y que durará hasta el año 1460. Samsuiluna tampoco pudo impedir que en el norte, la ciudad de Aššur se sublevase y pudiese conseguir su independencia por medio de un tal Adasi, hijo de nadie (etiqueta que aludía a un usurpador) pero que pasará a ser la persona que pondría fin a la subordinación de Aššur.

A pesar de todos estos reveses, Samsuiluna sí pudo lograr el control del Éufrates medio al vencer al rey Muti-Huršana, rey de Hुana (Anat).

Al final de su reinado Samsuiluna perdería todo el país de Sumer, los territorios del alto Tigris, manteniendo los territorios del medio Éufrates, es decir, volvió a sus fronteras originales, las del país de Acad. De todo lo que Hammurapi había conseguido, Samsuiluna sólo pudo mantener el valle del Diyala (país de Warum o Ešnunna).

No se conocen textos de las ciudades meridionales como Ur, Uruk y Larsa, después del décimo año de reinado de Samsuiluna. Sin embargo, para un tiempo tan posterior como el

\footnotetext{
${ }^{3}$ Samsu (forma amorrita de Šamaš, el dios-sol) es nuestro dios. El texto transliterado se encuentra recogido por Borger 1979 I: 51-52. El texto cuneiforme en escritura neoasiria según el ejemplar de Berlín en Borger II: 315. ${ }^{4}$ Ungnad 1938 II:182-192.
} 
vigésimo noveno año de Samsuiluna, en Nippur e Isin aún se reconocía la autoridad del rey babilonio, pero a partir de ese momento también desaparecen dicha información documental.

Lo cierto es que el imperio babilonio bajo el reinado de Samsuiluna dejó de controlar la parte sur de la ciudad de Nippur, la vida tanto urbana como rural continúa pacíficamente, si bien con ocasionales problemas de índole económico, en los centros septentrionales: Sippar, Kiš, Borsippa, Dilbat y en la propia Babilonia.

No podemos saber de una manera fidedigna si las ciudades meridionales bien tomadas o bajo influencia casita perdieron su identidad. La ausencia de documentación deja un vacío sobre cómo fue el paso del poder entre los reyes de la Primera Dinastía de Babilonia y la Dinastía Casita.

\section{La presencia casita}

Como muestran las fuentes a lo largo de la historia mesopotámica, la vida de los pueblos nómadas supuso una presencia constante que amenazaban en mayor o menor medida a las sociedades sedentarizadas. El pueblo casita constituía una excepción de estilo de vida con respecto a otros, debido a sus campamentos, organizados tribalmente y de origen oriental. La mayor parte de la información sobre la presencia casita ha sido reunida por B. Landsberger 5 . Este pueblo lingüísticamente está asociado a otros pueblos de la zona de los Zagros como los guti y elamitas. Las incursiones desde las montañas eran breves y, a excepción de los casitas, ninguna tribu montañosa pudo erigirse en dinastía asentada en la llanura.

\section{Cuadro descriptivo de la tablilla}

El texto cuneiforme que contiene la pieza presenta las siguientes características:

\section{Objeto: tablilla \\ Mineral: $\quad$ piedra caliza \\ Dimensiones: $\quad \mathbf{1 7 , 5}$ x 8,7 (cm) \\ Procedencia: desconocida \\ Museo: $\quad$ Vorderasiatischen Museum \\ $\mathrm{N}^{\mathrm{o}}$ Catalogo: $\quad$ VA $2645=$ E4. 3.7.5.1 A \\ Comentario: Consta de cuatro columnas. La primera contiene 20 líneas, las tres restantes 21. En total 83 líneas.}

Bibliografía: WINCKLER, H.-BÖHDEN, A. (1892), ABK n 74 (ej. 1, copia).;WINCKLER, H. (1892) KB3/1 pp.130-133 Samsu-iluna (ej. 1, edición).; KING, L. W. (1898-1900), LIH no 97 (ej. 2, copias; ej(s). 1-2 edición).; UNGNAD, A. (1907), VAS 1 n 33 (ej. 1, copia).; F. JEAN, (1923) Le Milieu Biblique pp. 110-111 (traducción).; EDZARD, D. O. (1957) Zwischenzeit pp. 124-125 (estudio).; BORGER, R. (1963), BAL ${ }^{1} 2$ pp. 47-48 (copia en escritura neo-asiria, transliteración y estudio); MATTHEWS, L. P. First Dynasty of Babylon pp. 187-200 (edición).; BORGER, R. (1963), BAL ${ }^{2} 1$ pp. 51-52 (copia en escritura neoasiria, transliteración y estudio).; I. KÄRKI, (1983), SAKAZ 2 pp. 19-25 (edición).; ZAMUDIO R. J. (2002) pp. 16-17 (transliteración) y pp. 287-288 (copia cuneiforme).

\footnotetext{
${ }^{5}$ Landsberger 1954: 31-45, 47-73, 106-133. Una revisión de la misma en Brinkman 1976-1980: 464-473. Algunas aportaciones en De Smet 1990: 1-19.
} 


\title{
5. Transliteración y traducción
}

\section{INSCRIPCIÓN $\mathbf{A}^{6}$}

\author{
Col. I (ll.1-20) \\ 1) $\quad i-n u{ }^{\mathrm{il}} \operatorname{Anum}\left({ }^{\mathrm{d}} \mathrm{AN}\right){ }^{7 \mathrm{il}} \operatorname{Ellil}\left({ }^{\mathrm{d}} \mathrm{EN} . \mathrm{LIL}\right)^{8}$ \\ Cuando los dioses Anu (y) Enlil, \\ 2) $\quad \check{a} a r-r u \check{s} a$ šamê(AN) ù erșetim(KI) \\ los reyes de los cielos y la tierra, \\ 3) $\quad a-n a{ }^{\text {il }} \operatorname{Marduk}\left({ }^{\mathrm{d}} \mathrm{AMAR} . \mathrm{UTU}\right)^{9}$ \\ al dios Marduk, \\ 4) $\quad[m \bar{a} r] \operatorname{im}([\mathrm{DU}] \mathrm{MU}) \mathrm{re-eš-ti-im/} \mathrm{ša} \dot{E}^{-} a^{10}$ \\ hijo primogénito del dios $\mathrm{Ea}$, \\ 5) ha-di-iš ip-pa/-al-sú-šum ${ }^{11}$ \\ alegremente, lo contemplaron. \\ 6) be-lu-ut ki-ib-ra-at/ ar-ba-im ${ }^{12}$ \\ El señorío de las cuatro regiones del mundo \\ 7) i-ti(di??)-nu-šum ${ }^{13}$ \\ le dieron a él. \\ 8) in ${ }^{\text {il } A-n u n-n a-k i^{14}}$ \\ Entre los dioses Anunnaki,
}

\footnotetext{
${ }^{6}$ Borger 1979 I: 51-52; II: 315 (en escritura neo-asiria según el ejemplar de Berlín).

${ }^{7}$ Desde antaño hubo una relación estrecha entre el panteón mesopotámico y el orden político humano. El éxito político supone entre otras consideraciones el favor divino. El éxito político iba estrechamente unido al favor divino. El dios An (Anu(m) en acadio) el $<<$ dios-cielo $>>$, era la divinidad más elevada tanto en sentido propio como figurado. Considerado el "rey de los dioses" y "rey de los Anunnaki" (los grandes dioses) apenas influye en los asuntos de los hombres. Relegado al firmamento, se considera una divinidad lejana y mal definida, pero como se aprecia en el texto no deja de ocupar un sitio preeminente.

${ }^{8} \mathrm{Si}$ Anu(m) es el "rey de los dioses", Enlil el $<<$ dios-aire $>>$ es el auténtico dios supremo que pasó a ser el jefe de la nueva representación de divinidades sumerias y acadias. Es él quien elige a los soberanos y del mismo modo que las órdenes de un monarca garantizan la supervivencia y la prosperidad de su población. Tenía su centro cultual en la ciudad de Nippur y su templo era conocido por el nombre de Ekur.

${ }^{9}$ El dios Marduk pasó a ser el dios supremo del panteón babilónico durante la Primera Dinastía Amorrea, la divinidad protectora de Babilonia. Era considerado el líder de los dioses y el garante de la realeza.

${ }^{10}$ En sumerio Enki. Dios de las aguas dulces, de la sabiduría y de los sortilegios. Tenía su centro cultual en la ciudad de Eridu.

${ }^{11}$ Pret. (N) de palāsu(m) "mirar", "contemplar", "dirigir la mirada" $3^{\mathrm{a}}$. pers. pl. Aunque tiene sentido pasivo tiene significado activo más dativo -šum en alusión al dios Marduk de la línea $\mathrm{n}^{\circ} .3$.

${ }^{12}$ El primer testimonio de este título real es acadio y está atestiguado con Narâm-Sin. Es difícil conocer el origen del título. Cf. López Montero, Pino Cano, Torres Torres 2014: 47 n.(6).

${ }^{13}$ Pret. (G) de nadānu(m) (i) "dar", "entregar" 33. pers. pl.

${ }^{14}$ En el corpus de textos sumerios, Anunna. En acadio (Anunnaki, Anunnaku). Considerados un grupo de dioses superiores en el panteón mesopotámico, pero también aparecen designando al panteón de una ciudad estado en particular, por ejemplo, los Anunna de Eridu, o los Anunna de Lagaš. Su nombre aparece escrito en sumerio bajo las formas: ${ }^{\mathrm{d}} \mathrm{a}-n u n,{ }^{\mathrm{d} a-n u n-n a},{ }^{\mathrm{d}} \mathrm{a}-n u n-n a-\mathrm{ke}_{4}-\mathrm{ne},{ }^{\mathrm{d}} \mathrm{a}-n u n-\mathrm{ke}_{4}-n e$. En acadio ${ }^{\mathrm{d}} a-n u n-n a k i, e-n u n-n a-$ $k i, e-n u-u k-k i,{ }^{\mathrm{d}} e-n u-u k-k i$. El dios Enki después de poner al dios sol Utu a cargo del universo, les asigna a los Anunnaki, considerados dioses de rango inferior, un lugar para vivir en la tierra. Enki les prohíbe regresar al $a b z u$, considerado la morada de los dioses. Ante las quejas de éstos y para liberarlos de los trabajos, Enki decidió crear la humanidad. Cf. Falkenstein 1965: 127-140; Burkhart 1965: 141-158; Katz 2003: 403. No está claro cuántos dioses y qué dioses incluye este término. Un texto habla de los "cincuenta Anunna de Eridu". Para este aspecto Cf. Falkenstein 1965: 130; Edzard 1965: 42. Además vid. Bottèro 2001: 79, 134, 167 y 169.
} 
9) $\quad \check{s} u$-ma-am și-ra-am/ i-bí-ù-šu $u^{15}$

llamaron su nombre excelso.

10) $\quad B[\bar{a}]$ bilam(KÁ.DINGIR.RA) ${ }^{\mathrm{ki} 16}$

(En cuanto a) Babilonia,

11) išd

$\mathrm{su}(\mathrm{s})$ cimiento(s) como los cielos y la tierra

12) ú-ki-in-nu-šum ${ }^{17}$

fijaron para él.

13) i-nu-šu $\left.{ }^{i l} \operatorname{Marduk(}{ }^{\mathrm{d} A M A R . U T U}\right)$

Entonces el dios Marduk,

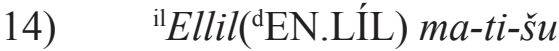

el dios Enlil, (de) su país,

15) ilum(DINGIR) ba-ni ne-me-qí-im

el dios creador de sabiduría,

16) a-na Sa-am-su-i-lu-na

a Samsuiluna,

17) $5 \check{s} a ̀ r(L U G A L) ~ l a-l e-s ̌ u / i a-t i$

el rey de su abundancia, a mí,

18) naphar( ̌́U.NIGÍN) ma-ta-tim

la totalidad de los países

19) a-na re-ia-im ${ }^{18}$ i-din-nam

me dio para gobernar,

20) $[m \bar{a}] s([\mathrm{~K}] \mathrm{ALAM})-$ sú a-bur-ri/ $\check{s} u-u r-b u-s a-a m^{19}$

asentar su país en prados,

\section{Col. II (ll.1-21)}

1) ni-ši-šu ra-ap-ša-tim

sus gentes numerosas

2) in $\check{s} u$-ul-mi-im

en bienestar (y)

3) a-na da-ar i-tar-ra-am ${ }^{20}$

para siempre guiar

4) $\quad r a-b i-i s ̌ s ~ l u-u ́-w a /-e-r a-a n-n i^{21}$

firmemente me ordenó.

5) Sa-am-su-i-lu-na $a^{22}$

Yo, Samsuiluna,

6) ร̌arrum(LUGAL) da-núm

rey poderoso,

\footnotetext{
capital del imperio de Hammurapi.

${ }^{17}$ Pret. (D) de kânu(m) (-u-) "establecer", "fijar" $3^{\mathrm{a}}$. pers. pl.

${ }^{18}$ Inf. $(\mathrm{G})$ en Genitivo de $r e$ ' $\hat{u}(m) / r \bar{e} y \hat{u}(m)$ "apacentar", "gobernar".

${ }^{19}$ Inf. ( $\left.\breve{S}\right)$ de $\operatorname{rabāṣu}(m)$ (i) "hacer yacer = asentar".

${ }^{20}$ Inf. (Gtn) en Acusativo de warû(m) (-u;-i) "guiar", "conducir", "llevar".

${ }^{21}$ Pret. (D) de wa'āru(m)/wâru(m) (-a-) "salir" + ni ac. $1^{\text {a }}$ pers.

${ }^{22}$ Vidal 2014: 42.
}

${ }^{15}$ Pret. $(\mathrm{G})$ de $n a b \hat{u}(m)$ (-i) sin contraer las vocales (ítù) "llamar", “nombrar", "elegir" $3^{\mathrm{a}}$. pers. pl.

${ }^{16}$ Nombre que hace referencia tanto a la ciudad de Babilonia como a sus habitantes. (en sumerio KÁ.DINGIR. RA.KI). La ciudad fue conocida con el nombre acadio de bab ilim/ili, "Puerta del dios/de los dioses". Fue la 
7) $\quad$ šàr Bābilim(KÁ.DINGIR.RA) ${ }^{\mathrm{ki}}$

rey de Babilonia,

8) క̌arrum mu-uš-te/-eš-mi

el rey (que) ha sometido

9) ki-ib-ra-at

regiones del mundo

10) ar-ba-im $a-n a-k u$

las cuatro, yo

11) in e-mu-uq/ ra-ma-ni-ia

con la fuerza de mi persona,

12) in igigallī(IGI.GÁL)-ia/ ra-bi-im

con mi gran sabiduría,

13) $d \bar{u} r(\mathrm{BAD}) \operatorname{Dimat}(\mathrm{AN} . Z A . G A ̀ R)-{ }^{\mathrm{i}} \operatorname{Ellil}\left({ }^{\mathrm{d}} \mathrm{EN} . L I ́ L\right){ }^{(\mathrm{ki})}$

la muralla Dimat-Enlil,

14) $\quad a-n a{ }^{\text {il Nin-mah }}$

a la diosa Ninmah,

15) ummin(AMA) ba-ni-ti-ia

madre, la que me ha engendrado,

16) $\quad d \bar{u} r(\mathrm{BAD}) P \grave{a}-d a^{\mathrm{ki}}$

la muralla Pada,

17) a-na ${ }^{\text {il }}$ Adad( $(\mathrm{d}$ IŠKUR) re-și-ia

para el dios Adad, el que me ha ayudado,

18) $\quad d \bar{u} r(\mathrm{BAD}) L a-g a-b a^{\mathrm{ki}}$

la muralla Lagaba,

19) $\quad a-n a{ }^{\mathrm{il}} \operatorname{Sin}(\mathrm{EN} . \mathrm{ZU}) / \operatorname{ilim}(\mathrm{DINGIR}) b a-n i-i a$

para el dios Sin, el que me ha creado,

20) $d \bar{u} r(\mathrm{BAD}){ }^{(\mathrm{uru})} I a-b u-{\text { š } u m^{\mathrm{ki}}}^{2}$

la muralla Iabušum,

21) $\quad a-n a{ }^{\text {il LUGAL.GIŠ.ásal(A.TU.GAB.LIŠ) }}$

para el dios Šar-ṣabatim,

Col. III (ll.1-21)

1) $\quad m u$-šar-bí-ùl šar-ru-ti-ia

el que ha engrandecido mi realeza,

2) $\quad d \bar{u} r(\mathrm{BAD}) a l(\mathrm{URU}) G u-l a-b a_{8}^{\mathrm{ki}}$

la muralla Gulaba

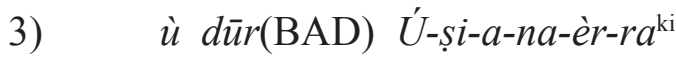

y la muralla Uși-ana-Erra

4) $\quad a-n a{ }^{\text {il }} \operatorname{Nergal}\left(\mathrm{NÈ} . \mathrm{ERI}_{11}\right.$.GAL)

para el dios Nergal,

5) ha-ti a-a-bi-ia

el que ha derribado a mis enemigos.

6) 6 dūrānn̄(BAD.MEŠS) ra-bu-tim/ $\check{s} u-n u-t i$

Esas seis grandes murallas

7) $\quad \check{a}$ Su-mu-la-il

que Sūmû-la-Il,

8) $\quad a-b i$ ra-bu-um

mi antepasado, 
9) $\quad a-b i \quad a-b i-i a$

padre de mi padre,

10) h̆a-am-šum i-pu-šu

(mi) quinta (generación) había construido

11) in la-bi-ru-ti-šsu-nu

por su vejez (de las murallas),

12) in ra-ma-ni-šu-nu

por sí mismas

13) up-ta-as-sí-su-ma

se habían agrietado.

14) in $l i-i b-b u$ ITU.2.KAM

En el transcurso de dos meses,

15) $\quad \operatorname{libitta}\left(\mathrm{SIG}_{4}\right)$-šu-nu al-bi-in

yo modelé sus ladrillos

16) $\quad r a-b i-i s ̌$ e-pu-uś

(y) grandemente construí.

17) re-ši-šu-nu ki-ma/ šadîm(SA.TU)-im ú-ul-li

Yo alcé su cima como una montaña.

18) naphar(ŠU.NIGÍN) ma-ta-tim išdì(SUHUŠ)-ši-na/ ú-ki-in Al conjunto de los países sus cimientos hice firmes.

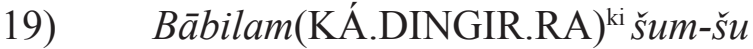

A la (ciudad) de Babilonia, su nombre

20) lu ú-ṣi-ir

ciertamente, yo engrandecí

21) in ki-ib-ra-at/ ar-ba-im

en las cuatro regiones del mundo,

Col. IV (ll.1-21)

1) lu ú-ša-tir-šu

ciertamente, yo lo enaltecí.

2) $\quad$ pu-luh-ti melemmī(ME.LÁM)/ šar-ru-ti-ia

El temor del resplandor de mi realeza

3) pa-at šamê(AN) ì er-șe-tim

las fronteras de cielos y tierra

4) lu ik-tum

ciertamente, cubrió.

5) $\quad a-n a ~ s ̌ u-a-t i$ ilū rabûtum(DINGIR.GAL.GAL)

Por esto, los dioses grandes

6) in bu-ni-šu-nu na-aw-ru-tim

con sus rostros resplandecientes,

7) lu ip-pa-al-su-nim

ciertamente, me miraron.

8) ba-la-țum ša ki-ma/ ${ }^{\mathrm{il}} \operatorname{Sin}(\mathrm{EN} . Z \mathrm{ZU} / Z \mathrm{ZUN})$

Una vida que como el dios Sîn

9) wa-ar-hi-ša-am

mensualmente

10) ú-te-ed-de-šu

se renueva, 
11) re-iu-ut ki-ib-ra-at/ ar-ba-im

el pastoreo de las cuatro regiones del mundo,

12) in šu-ul-mi-im

en bienestar

13) a-na da-ar e-pé-ša-am

para siempre construir

14) $\quad n i-i z-m a-a t / l i-i b-b i-i a$

los deseos de mi corazón,

15) ki-ma ilim(DINGIR) ka-ša-dam

como un dios lograr

16) $u_{4}-m i-s ̌ a-a m$ in re-ši-in/ e-li-a-tim

diariamente, con la cabeza alta (y)

17) in ri-ša-a-tim

con júbilo

18) ù hu-ud-li-ib-bi-im

y alegría de corazón,

19) a-ta-al-lu-kam

caminar una y otra vez

20) a-na ̌̌e-ri-ik-tim

como un don,

21) lu iš-ru-ku-nim

ciertamente me regalaron.

\section{Traduccion}

Cuando los dioses Anu (y) Enlil, los reyes de los cielos y la tierra, al dios Marduk, hijo primogénito del dios Ea, alegremente, lo contemplaron, el señorío de las cuatro regiones del mundo le dieron a él. Entre los dioses Anunnaki, llamaron su nombre excelso. (En cuanto a) Babilonia, su(s) cimiento(s) como los cielos y la tierra fijaron para él. Entonces el dios Marduk, el dios Enlil de su país, el dios creador de sabiduría, a Samsuiluna, el rey de su abundancia, a mí, la totalidad de los países me dio para gobernar, asentar su país en prados, sus gentes numerosas en bienestar (y) para siempre guiar firmemente me ordenó. Yo, Samsuiluna, rey poderoso, rey de Babilonia, el rey (que) ha sometido regiones del mundo, las cuatro, con la fuerza de mi persona, con mi gran sabiduría, la muralla Dimat-Enlil, a la diosa Ninmah madre, la que me ha engendrado, la muralla Pada, para el dios Adad, el que me ha ayudado, la muralla Lagaba, para el dios Sin, el que me ha creado, la muralla Iabušum, para el dios Šar-șabatim, el que ha engrandecido mi realeza, la muralla Gulaba y la muralla Ușiana-Erra para el dios Nergal, el que ha derribado a mis enemigos. Esas seis grandes murallas que Sūmû-la-Il, mi antepasado, padre de mi padre, (mi) quinta (generación) había construido por su vejez (de las murallas), por sí mismas se habían agrietado. En el transcurso de dos meses, yo modelé sus ladrillos (y) grandemente construí. Yo alcé su cima como una montaña. Al conjunto de los países sus cimientos hice firmes. A la (ciudad) de Babilonia, su nombre ciertamente, yo engrandecí en las cuatro regiones del mundo, ciertamente, yo lo enaltecí. El temor del resplandor de mi realeza las fronteras de cielos y tierra ciertamente, cubrió. Por esto, los dioses grandes con sus rostros resplandecientes, ciertamente, me miraron. Una vida que como el dios Sîn mensualmente se renueva, el pastoreo, de las cuatro regiones del mundo en bienestar para siempre construir los deseos de mi corazón, como un dios lograr diariamente, con la cabeza alta (y) con júbilo y alegría de corazón, caminar una y otra vez como un don, ciertamente me regalaron. 
7. Arquitectura defensiva: construcción y restauración de murallas recogidas en los $<<$ nombres de año $>>$ desde Sumuabum hasta Samsuiluna

El empleo a comienzos del segundo milenio a.C. en las fuentes históricas babilonias de dar título a los nombres de años, tuvo como objetivo básico el cálculo del tiempo. No obstante, el sistema empleado por estos monarcas de origen amorrita, no se llevó a cabo por medio de un de un epónimo particular que llevaba el nombre propio de un alto cargo de la administración, sino más bien, hacía referencia a un evento que destacase sobre otros. Estos nombres de años se caracterizan por ser breves y concisos en cuanto a los hechos que registra. Así tenemos la siguiente tabla de construcciones y restauraciones de murallas (bàd/ dûru $)^{23}$ llevadas a cabo por el fundador de la primera dinastía de Babilonia, Sumuabum, pasando por Samsuiluna y los que le sucedieron en el gobierno. Con Hammurapi, el imperio babilonio alcanzó su máxima expansión territorial. La estrategia militar llevada a cabo por su hijo Samsuiluna no podía sino limitarse a la consolidación del territorio heredado por su progenitor, para lo que fue precisa una política fronteriza constante y rigurosa.

\begin{tabular}{|c|c|c|c|}
\hline Rey & $\begin{array}{l}\text { Año de Reinado } \\
\text { Transliteración en } \\
\text { sumerio }\end{array}$ & Acontecimiento & Fuente \\
\hline \multirow[t]{5}{*}{$\begin{array}{c}\text { Sumuabum } \\
(1894-1881)\end{array}$} & $\begin{array}{l}\text { 1b (1894) } \\
\text { mu bàd gal ká-din- } \\
\text { gir-ra }{ }^{\{k i\}} \text { ba-dù }\end{array}$ & $\begin{array}{c}\text { Año en que se } \\
\text { construyó la gran } \\
\text { muralla de la ciudad } \\
\text { de Babilonia }\end{array}$ & R1A 2175,1 \\
\hline & $\begin{array}{c}\text { 3a }(1892) \\
\text { mu bàd e-li-ip }{ }^{\{\mathrm{ki}\}} \\
\text { ba-dab }\end{array}$ & $\begin{array}{c}\text { Año en que se } \\
\text { construyó la muralla } \\
\text { de Ellipi }\end{array}$ & RlA 2175,3 \\
\hline & $\begin{array}{l}9(1886) \\
\text { mu bàd dil-bat }{ }^{\{k i\}} \\
\text { ba-dù }\end{array}$ & $\begin{array}{c}\text { Año en que se } \\
\text { construyó la muralla } \\
\text { (de la ciudad) de } \\
\text { Dilbat }\end{array}$ & RlA 2175,9 \\
\hline & $\begin{array}{c}10 \text { (1885) } \\
\text { mu bàd-an-ne kišs }{ }^{\{k i\}} \\
\text { mu-un-na-dím-ma }\end{array}$ & $\begin{array}{l}\text { Año en que (Sumu- } \\
\text { abum) hizo para } \\
\text { Kiš su muralla } \\
\text { (alcanzando) el cielo }\end{array}$ & $?=\mathrm{R} 1 \mathrm{~A} 2175,10 ?$ \\
\hline & $\begin{array}{c}11 \text { (1884) } \\
\text { mu ús-sa bàd-an-ne } \\
\text { kišs }{ }^{\{\mathrm{ki}\}} \text { mu-un-na- } \\
\text { dím-ma }\end{array}$ & $\begin{array}{l}\text { Año después del } \\
\text { año en que (Sumu- } \\
\text { abum) hizo para } \\
\text { Kiš su muralla } \\
\text { (alcanzando) el cielo }\end{array}$ & R1A 2175,11 \\
\hline
\end{tabular}

${ }^{23}$ El término sumerio bàd se ha transcrito en negritas en este trabajo. 


\begin{tabular}{|c|c|c|c|}
\hline \multirow[t]{3}{*}{$\begin{array}{c}\text { Sumu-la-Il } \\
(1881-1845)\end{array}$} & $\begin{array}{c}5(1876) \\
\text { mu bàd gal tin-tir } \\
\text { / ká-dingir-ra }^{\{\mathrm{ki}\}} \text { ba- } \\
\text { dù }\end{array}$ & $\begin{array}{l}\text { Año en que se } \\
\text { construyó la gran } \\
\text { muralla de Tintir (y) } \\
\text { de Babilonia }\end{array}$ & RlA 2165,19 \\
\hline & $\begin{array}{l}6(1875) \\
\text { mu ús-sa bàd gal } \\
\text { tin-tir }{ }^{\{\mathrm{ki}\}} / \text { ká-din- } \\
\text { gir-ra }^{\{\mathrm{ki}\}} \text { ba-dù }\end{array}$ & $\begin{array}{l}\text { Año tras año se } \\
\text { construyó la gran } \\
\text { muralla de Tintir (y) } \\
\text { de Babilonia }\end{array}$ & R1A 2165,20 \\
\hline & $\begin{array}{c}19(1862) \\
\text { mu bàd-an-ne kišs }{ }^{\{\mathrm{ki}\}} \\
\text { ba-gul }\end{array}$ & $\begin{array}{c}\text { Año en que se } \\
\text { destruyó el muro de } \\
\text { la ciudad (llegando } \\
\text { hasta) el cielo de } \\
\text { Kiš }\end{array}$ & RlA 2165,33 \\
\hline & $\begin{array}{l}20(1861) \\
\text { mu bàd ka-zal-lu\{ki\} } \\
\text { ba-gul ùéren-bi }\{g i s ̌\} \\
\text { tukul ba-sìg }\end{array}$ & $\begin{array}{c}\text { Año en que la } \\
\text { muralla de la } \\
\text { ciudad de Kazallu } \\
\text { fue destruida y su } \\
\text { ejército fue herido } \\
\text { por armas }\end{array}$ & RlA 2165,34 \\
\hline & $\begin{array}{c}27(1854) \\
\text { mu bàd gú-du }{ }_{8}-\mathrm{a}^{\{\mathrm{ki} i} \\
\text { ù an-za-gàr-ur-gi }{ }_{7}{ }^{\{\mathrm{ki}\}} \\
\text { ba-dù }\end{array}$ & $\begin{array}{l}\text { Año en que se } \\
\text { construyeron las } \\
\text { murallas de Kutha y } \\
\text { Anzagar-urgi }\end{array}$ & R1A 2165,41 \\
\hline & $\begin{array}{c}\text { 29a (1852) } \\
\text { mu bàd zimbir }{ }^{\{\mathrm{ki}\}} \\
\text { su-mu-la-èl lugal } \\
\text { ba-dù }\end{array}$ & $\begin{array}{c}\text { Año en que } \\
\text { Sumu-la-El, el rey } \\
\text { construyó la muralla } \\
\text { de la ciudad de } \\
\text { Sippar }\end{array}$ & R1A 2165,43 \\
\hline & $\begin{array}{c}31(1850) \\
\text { mu bàd gal ha-bu- } \\
\text { uz } \\
\text { \{ki\} ba-dù }\end{array}$ & $\begin{array}{c}\text { Año en que se } \\
\text { construyó la gran } \\
\text { muralla de la ciudad } \\
\text { de Habuz }\end{array}$ & R1A 2165,45 \\
\hline & $\begin{array}{c}\text { 36b (1845) } \\
\text { mu su-mu-la-èl bàd } \\
\text { dal-bat }{ }^{\{k i\}} / \text { dul-ba- } \\
\text { tum ba-dù }\end{array}$ & $\begin{array}{c}\text { Año en el que } \\
\text { Sumu-la-El } \\
\text { construyó la muralla } \\
\text { de la ciudad de } \\
\text { Dilbat }\end{array}$ & CT 8 44b:30 \\
\hline
\end{tabular}




\begin{tabular}{|c|c|c|c|}
\hline $\begin{array}{c}\text { Sabium } \\
(1845-1831)\end{array}$ & $\begin{array}{c}\text { 2b (1843) } \\
\text { mu su-mu-la-èl bàd } \\
\text { dal-bat }{ }^{\{\mathrm{ki}\}} / \text { dul-ba- } \\
\text { tum ba-dù }\end{array}$ & $\begin{array}{c}\text { Año en el que } \\
\text { Sabium construyó } \\
\text { la muralla de Kar- } \\
\text { Šamaš }\end{array}$ & BE 6.110 \\
\hline & $\begin{array}{l}3 \text { (1842) } \\
\text { mu ús-sa bàd kar-\{d\} } \\
\text { utu }^{\{k i\}} \text { sà-bu-um ba- } \\
\text { dù }\end{array}$ & $\begin{array}{c}\text { Año tras año } \\
\text { Sabium construyó la } \\
\text { muralla de la ciudad } \\
\text { de Kar-Šamaš }\end{array}$ & R1A 2 176, 54 \\
\hline & $\begin{array}{l}12(1833) \\
\text { mu bàd ka-zal-lu } \\
\text { ba-hiki }\end{array}$ & $\begin{array}{l}\text { Año en que la } \\
\text { muralla de Kazallu } \\
\text { fue destruida }\end{array}$ & RlA 2 176, 62 \\
\hline \multirow[t]{5}{*}{$\begin{array}{c}\text { Apil-Sîn } \\
(1831-1813)\end{array}$} & $\begin{array}{c}\text { 1c (1831) } \\
\text { mu a-pil-\{d\}en.zu } \\
\text { lugal-e bàd bar-sí- } \\
\text { pa }^{\{k i\}} \text { ba-dù }\end{array}$ & $\begin{array}{l}\text { Año en que Apil- } \\
\text { Sîn, el rey constru- } \\
\text { yó la muralla de la } \\
\text { ciudad de Barsipa }\end{array}$ & $\begin{array}{c}\text { CT } 69 \text { II, 9; CT } 8 \\
29 b\end{array}$ \\
\hline & $\begin{array}{c}\text { 2b (1830) } \\
\text { mu bàd-an-na }{ }^{\{n a 4\}} \\
\text { za-gìn ká-dingir-ra }^{\{k i\}} \\
\text { a-pil-\{d\} en.zu mu-na- } \\
\text { dím }\end{array}$ & $\begin{array}{l}\text { Año en que Apil- } \\
\text { Sîn construyó } \\
\text { la muralla de la } \\
\text { ciudad de Babilonia } \\
\text { (alcanzando) el cielo } \\
\text { (y brillando como) } \\
\text { lapislázuli }\end{array}$ & BM 81109 \\
\hline & $\begin{array}{c}4^{*}(1828) \\
\text { mu ús-sa ús-sa-a } \\
\text { bàd uru za-gìn kù } \\
\text { mu-dù? }\end{array}$ & $\begin{array}{l}\text { Segundo año } \\
\text { después del año } \\
\text { (el rey) hizo la } \\
\text { muralla de la ciudad } \\
\text { (de Babilonia) } \\
\text { (brillante) de } \\
\text { lapislázuli y metal }\end{array}$ & Iraq 48126 \\
\hline & $\begin{array}{c}\text { (1827) } \\
\text { mu a-pil-\{d\} } \\
\text { en.zu lugal-e bàd } \\
\text { nu-rim }{ }^{\{\mathrm{ki}\}} \text { ba-dù }\end{array}$ & $\begin{array}{l}\text { Año Apil-Sîn, el rey } \\
\text { construyó la muralla } \\
\text { de la ciudad de } \\
\text { Nurum }\end{array}$ & Iraq 48126 \\
\hline & $\begin{array}{c}\text { 11a (1819) } \\
\text { mu a-pil-\{d\} en.zu } \\
\text { lugal-e dur mu-ti }{ }^{\{\mathrm{ki}\}} \\
\text { ba-dù }\end{array}$ & $\begin{array}{l}\text { Año en que Apil- } \\
\text { Sîn construyó la } \\
\text { (muralla) de (la } \\
\text { ciudad) de Muti }\end{array}$ & $\begin{array}{c}\text { CT } 447 \mathrm{a}, 46+\mathrm{IM} \\
85922+85934\end{array}$ \\
\hline
\end{tabular}




\begin{tabular}{|c|c|c|c|}
\hline & $\begin{array}{l}\text { 11b (1819) } \\
\text { mu bàd mu-ti } \\
\text { ba-dù }\end{array}$ & $\begin{array}{c}\text { Año en que se } \\
\text { construyó la muralla } \\
\text { de Muti }\end{array}$ & IM $85922+95934$ \\
\hline & $\begin{array}{l}\text { 12a (1818) } \\
\text { mu un / bàd gú íd- } \\
\text { idigna a-pil } \\
\text { šu bí-in-zi / ki bí- } \\
\text { in-gi }\end{array}$ & $\begin{array}{l}\text { Año en que Apil- } \\
\text { Sîn se instaló / } \\
\text { restableció el muro } \\
\text { en las orillas del } \\
\text { Tigris }\end{array}$ & $\begin{array}{c}\text { CT } 45 \text { 101, BM } \\
82412\end{array}$ \\
\hline & $\begin{array}{c}\text { 17c (1815) } \\
\text { mu ú-pí-e } \text { eki\} a-pil }^{-\{\mathrm{d}\}} \\
\text { en.zu ba-dù }\end{array}$ & $\begin{array}{c}\text { Año Apil-Sîn } \\
\text { construyó (la } \\
\text { muralla de la ciudad } \\
\text { de) Upi }\end{array}$ & BM 22713 \\
\hline \multirow[t]{6}{*}{$\begin{array}{l}\text { Sîn-Muballit } \\
(1813-1793)\end{array}$} & $\begin{array}{c}\text { 1b (1813) } \\
\text { mu \{d\}en.zu-mu- } \\
\text { ba-lí-it lugal-e bàd } \\
\text { syar-ba-tum }{ }^{\{k i\}} \text { mu- } \\
\text { dù }\end{array}$ & $\begin{array}{l}\text { Año Sîn-Muballit, } \\
\text { el rey construyó la } \\
\text { muralla de la ciudad } \\
\text { de Si'arbatum }\end{array}$ & RlA 2166,83 \\
\hline & $\begin{array}{l}10(1804) \\
\text { mu bàd-\{d\} } \mathrm{en.zu-} \\
\text { mu-ba-lí-it ba-dù }\end{array}$ & $\begin{array}{l}\text { Año en que Sin- } \\
\text { muballit construyó } \\
\text { la muralla de (?) }\end{array}$ & R1A 2 166, 92 \\
\hline & $\begin{array}{c}11 \text { (1803) } \\
\text { mu \{d\}en.zu-mu- } \\
\text { ba-lí-it lugal-e bàd } \\
\text { már-da }{ }^{\{k i\}} \text { mu-un- } \\
\text { dù-a }\end{array}$ & $\begin{array}{l}\text { Año en que Sin- } \\
\text { muballit, el rey } \\
\text { construyó la muralla } \\
\text { de la ciudad de } \\
\text { Marad (Kazallu) }\end{array}$ & R1A 2 166, 94 \\
\hline & $\begin{array}{c}14(1800) \\
\text { mu bàd érešs }{ }^{\{\mathrm{ki}\}}\{\mathrm{d}\} 30- \\
\text { mu-ba-lí-it ba-dù }\end{array}$ & $\begin{array}{l}\text { Año en que Sin- } \\
\text { muballit construyó } \\
\text { la muralla de la } \\
\text { ciudad de Ereš }\end{array}$ & R1A 2 166, 97 \\
\hline & $\begin{array}{c}17(1797) \\
\text { mu bàd uru } \\
\text { ba-si’um }\{k i\} \text { ba-dù }\end{array}$ & $\begin{array}{c}\text { Año que se } \\
\text { construyó la muralla } \\
\text { de la ciudad de } \\
\text { Bazûm }\end{array}$ & R1A 2166,100 \\
\hline & $\begin{array}{l}19(1795) \\
\text { mu \{d\}en.zu-mu-ba- } \\
\text { lí-it lugal-e bàd ši- } \\
\text { ra-mah'ski\} }\end{array}$ & $\begin{array}{l}\text { Año en que Sin- } \\
\text { muballit, el rey } \\
\text { construyó la muralla } \\
\text { de la ciudad de } \\
\text { Širamah }\end{array}$ & $\begin{array}{c}\text { YOS } 1396+\text { BM } \\
85922,85934\end{array}$ \\
\hline
\end{tabular}




\begin{tabular}{|c|c|c|c|}
\hline \multirow[t]{9}{*}{$\begin{array}{l}\text { Hammurapi } \\
(1793-1750)\end{array}$} & $\begin{array}{c}4(1790) \\
\text { mu bàd gal gá-gi } \\
\mathrm{a}^{\{\mathrm{ki}\}} / \mathrm{ga} \text {-gi-im gibil } \\
\text { bàdši-ra-maȟkik mu- } \\
\text { un-dù-a }\end{array}$ & $\begin{array}{c}\text { Año en que } \\
\text { (Hammurapi) } \\
\text { construyó la muralla } \\
\text { de la ciudad de } \\
\text { Širamah }\end{array}$ & $\begin{array}{c}\text { R1A } 2178,106 ; \mathrm{BM} \\
85449\end{array}$ \\
\hline & $\begin{array}{c}11(1783) \\
\text { mu bàd / uru ma-da } \\
\text { á-dam ra-pí-qum }{ }^{\{k i\}} \\
\text { ùša-li-bi }^{\{k i\}} \text { in-dab }\end{array}$ & $\begin{array}{l}\text { Año (Hammurapi) } \\
\text { cogió la muralla } \\
\text { de la ciudad? / } \\
\text { ciudad, la tierra } \\
\text { y el territorio de } \\
\text { Rapiqum y Šalibi }\end{array}$ & RlA 2178,113 \\
\hline & $\begin{array}{l}19(1775) \\
\text { mu bàd mah igi- } \\
\text { hur-sag-gá }\end{array}$ & $\begin{array}{l}\text { Año (Hammurapi } \\
\text { construyó) la } \\
\text { excelsa muralla } \\
\text { Igihursag(a) }\end{array}$ & R1A 2 179, 121 \\
\hline & $\begin{array}{l}\text { 21a (1773) } \\
\text { mu haa-am-mu-ra- } \\
\text { pi lugal-e bàd uru } \\
\text { ba-si’um }{ }^{\{k i\}} \text { ba-dù }\end{array}$ & $\begin{array}{c}\text { Año Hammurapi } \\
\text { construyó } \\
\text { Hammurapi la } \\
\text { muralla de la ciudad } \\
\text { de Bazûm }\end{array}$ & RlA 2179,123 \\
\hline & $\begin{array}{l}\text { 21b (1773) } \\
\text { mu bàdša ba-si-ú- } \\
\text { um }\end{array}$ & $\begin{array}{l}\text { Año (Hammurapi), } \\
\text { el rey construyó la } \\
\text { muralla de la ciudad } \\
\text { de Bazûm }\end{array}$ & BM 82128 \\
\hline & $\begin{array}{c}23(1771) \\
\text { mu uš bàd } \\
\text { zimbirík }^{\{k i\}}-\text { ra }\end{array}$ & $\begin{array}{c}\text { Año los cimientos } \\
\text { de la muralla de la } \\
\text { ciudad de Sippar (se } \\
\text { fijaron) }\end{array}$ & RlA 2179,125 \\
\hline & $\begin{array}{c}25(1769) \\
\text { mu bàd gal } \\
\text { zimbir }^{\{k i\}} \text {-ra }{ }^{\{\mathrm{d}\}} \text { utu } \\
\{\mathrm{d}\} \text { šè-ri } \\
\text { ba-da gul-la } \\
\text { ba-dù }\end{array}$ & $\begin{array}{l}\text { Año (Hammurapi) } \\
\text { reconstruyó la } \\
\text { muralla de la gran } \\
\text { destrucción de la } \\
\text { ciudad de Sippar }\end{array}$ & RlA 2179,127 \\
\hline & $\begin{array}{c}\text { 35a (1759) } \\
\text { mu ha-am-mu-ra- } \\
\text { pí lugal-e inim an } \\
\{\text { d\} en-líl-lá-ta bàd } \\
\text { má-rí }{ }^{\{k i\}} \text { ù bàd mà- } \\
\text { al-gi }{ }_{4}-\mathrm{a}\{k i\} \text { mu-un- } \\
\text { gul-la } \\
\end{array}$ & $\begin{array}{c}\text { Año en que } \\
\text { Hammurapi, el rey } \\
\text { por orden de An } \\
\text { y Enlil destruyó } \\
\text { las murallas de la } \\
\text { ciudad de Mari y } \\
\text { Malgium }\end{array}$ & R1A 2 180, 137 \\
\hline & $\begin{array}{l}\text { 35b (1759) } \\
\text { mu bàd má-rín }{ }^{k \mathrm{ki}\}} \mathrm{ù} \\
\text { sig }_{4}\{\mathrm{ki}\}\end{array}$ & $\begin{array}{l}\text { Año las murallas de } \\
\text { (la ciudad) de Mari } \\
\text { (y Malgium) }\end{array}$ & TCL 1101 \\
\hline
\end{tabular}




\begin{tabular}{|c|c|c|c|}
\hline & $\begin{array}{c}\text { 38a (1756) } \\
\text { mu ha-am-mu-ra- } \\
\text { pí lugal du }{ }_{11} \text {-ga an } \\
\text { \{d\}en-líl-bi-ta nam- } \\
\text { kù-zu }{ }^{\{d\}} \text { marduk-ke }{ }_{4} \\
\text { mu-un-na-an-sum- } \\
\text { ma-a bàd èš-nun- } \\
\text { na kki\} a gal-gal-la } \\
\text { mu-un-gul-la ... ki } \\
\text { íb-ta-zi / íb-ta-gi } \\
\end{array}$ & $\begin{array}{l}\text { Año Hammurapi, el } \\
\text { rey, por orden de An } \\
\text { y Enlil, por medio } \\
\text { de la sabiduría que } \\
\text { le dio a Marduk, } \\
\text { restauró la muralla } \\
\text { de la ciudad de } \\
\text { Ešnunna, destruida } \\
\text { por una inundación }\end{array}$ & BM 85386 \\
\hline & $\begin{array}{c}42(1752) \\
\text { mu ha-am-mu-ra-pí } \\
\text { lugal-e bàd gal gú } \\
\text { íd-idigna sag-bi hur- } \\
\text { sag-gim mu-un-íl-lá } \\
\text { kar- }{ }^{\{d\}} \text { utu }^{\{k i\}} \text { mu-bi } \\
\text { bí-in-sa }{ }_{4}^{-a} \text { ù bàd } \\
\text { ra-pí-qum }{ }^{\{k i\}} \text { gúíd- } \\
\text { buranun-ka mu-un- } \\
\text { dù-a }\end{array}$ & $\begin{array}{c}\text { Año en que } \\
\text { Hammurapi, el } \\
\text { rey, levantó como } \\
\text { una montaña, la } \\
\text { parte superior de } \\
\text { la muralla de la } \\
\text { gran ciudad en las } \\
\text { orillas del Tigris, } \\
\text { 1lamado Kar-Samaš } \\
\text { y construyó la } \\
\text { muralla de la ciudad } \\
\text { de Rapiqum en las } \\
\text { orillas del Éufrates } \\
\end{array}$ & R1A 2181,144 \\
\hline & $\begin{array}{c}\text { 43a (1751) } \\
\text { mu ha-am-mu- } \\
\text { ra-pí lugal-e zim- } \\
\text { bir }{ }^{\{k i\}} \text { uru-ù-ul } \\
\{d\} \text { utu-ke }- \text {-bàd-bi } \\
\text { sahar-gal-ta in-gar- } \\
\text { ra }\end{array}$ & $\begin{array}{c}\text { Año en que } \\
\text { Hammurapi, el rey } \\
\text { hizo el muro de } \\
\text { Sippar, la ciudad } \\
\text { eterna de Šamaš, de } \\
\text { grandes masas de } \\
\text { tierra }\end{array}$ & R1A 2181,145 \\
\hline & $\begin{array}{c}\text { 43b (1751) } \\
\begin{array}{c}\text { sanat eper zimbir }^{\{k i\}} \\
\text { iš-ša-ap-ku }\end{array}\end{array}$ & $\begin{array}{l}\text { Año en que la tierra } \\
\text { fue amontonada } \\
\text { para (la muralla de } \\
\text { la ciudad de) Sippar }\end{array}$ & PBS 737 \\
\hline \multirow[t]{2}{*}{$\begin{array}{l}\text { Samsuiluna } \\
(1749-1712) \\
\end{array}$} & & & \\
\hline & $\begin{array}{c}\text { 11a (1739) } \\
\text { mu sa-am-su-i-lu- } \\
\text { na lugal-e du }{ }_{11} \text {-ga } \\
\text { an }{ }^{\{d\}} \text { en-líl-lá-bi-ta / } \\
\{\mathrm{d}\} \text { en-líl-lá-bi-da bàd } \\
\text { úrí }^{\{k i\}} \text { ù unug }{ }^{\{k i\}} \text {-ga } \\
\text { mu-un-gul-la ugnim } \\
\text { ki-uri a-rá []-kam } \\
\{\text { gišs } \text { tukul-ta in-sìg-ge }\end{array}$ & $\begin{array}{c}\text { Año Samsuiluna, } \\
\text { el rey por orden de } \\
\text { An y Enlil destruyó } \\
\text { las murallas de } \\
\text { la ciudad de Ur y } \\
\text { Uruk y por enésima } \\
\text { vez golpeó por las } \\
\text { armas a las tropas de } \\
\text { Akkad }\end{array}$ & RlA 2183,156 \\
\hline
\end{tabular}




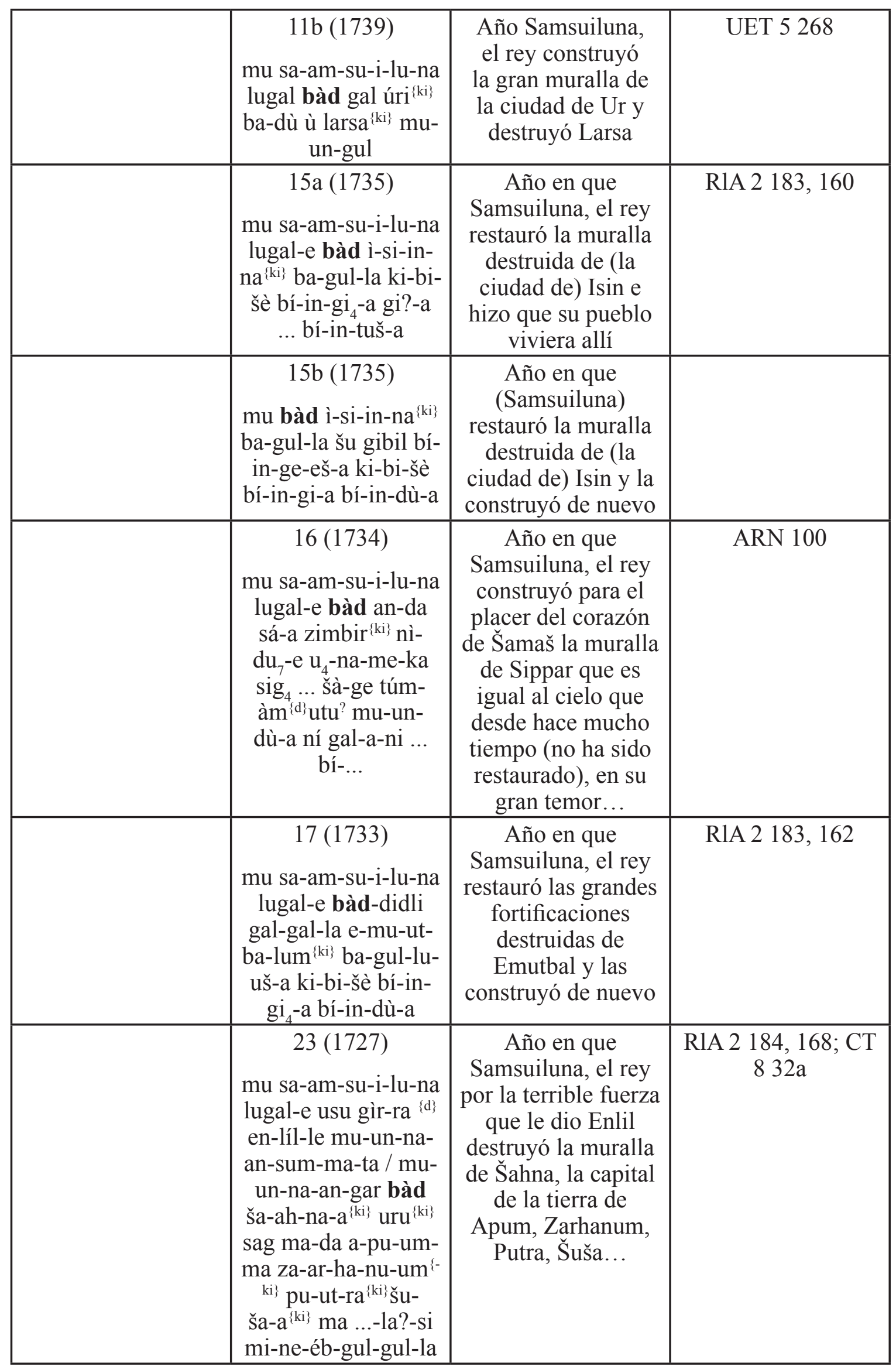




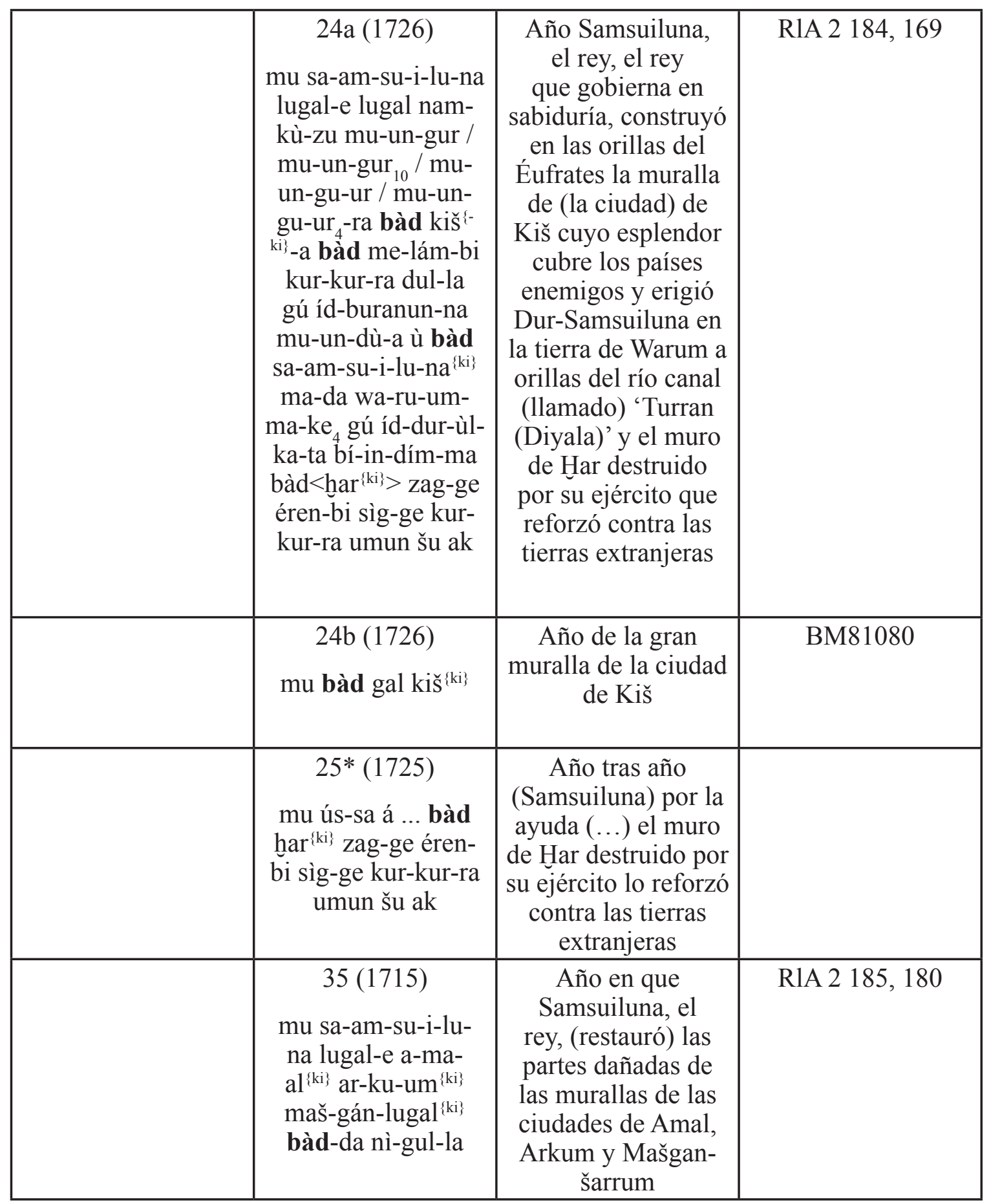

\section{Consideraciones finales}

El poema de Gilgameš narra que dicho rey de Uruk reparó y renovó sus muros que ya existían desde hacía mucho tiempo. De ello se deduce que una de las principales misiones que tenían los reyes mesopotámicos era mantener en perfecto estado las obras arquitectónicas de carácter defensivo. Samsuiluna, al igual que sus predecesores, da cuenta en los llamados $<<$ Nombres de año $>>$ los hechos más destacados acaecidos en ese intervalo de tiempo. Todos ellos dan gran importancia a la demolición, reconstrucción y construcción de murallas.

Durante el reinado de Hammurapi, el imperio babilonio alcanzó su máxima expansión territorial. Su tamaño fue de tal envergadura que por el contrario era difícil de defender ante la presión que ejercieron una serie de pueblos desde distintos lugares del imperio. La 
estrategia llevada a cabo por su hijo Samsuiluna no incidió en el mismo sentido y tan sólo se limitó a la consolidación del extenso territorio heredado por su progenitor para lo cual fue precisa realizar una política fronteriza constante y rigurosa. Las guerras frecuentes en que se vio envuelto Samsuiluna y sucesores dejó mermados sus esfuerzos para acometer la actividad económica que el imperio requería. Los $<<$ Nombres de año $>>$ dejan bien patente la construcción y reconstrucción de fortalezas y murallas en la mayoría de su reino para contener el avance del enemigo que acosaba desde distintos frentes.

Samsuiluna, al final de su gobierno dejó la monarquía más debilitada y su imperio bastante más reducido. Los errores cometidos por Samsuiluna fueron de carácter estructural y sus sucesores no tuvieron el suficiente fuste, ni la capacidad organizativa para mantener la "unidad" impuesta por Hammurapi. Samsuiluna debió hacer frente desde sus primeros años de reinado, a la presión ejercida por una serie de pueblos extranjeros, especialmente, los casitas (kašš $\hat{u})$ provenientes del suroeste del Irán. Según el escueto texto recogido en los $<<$ nombres de año $>>$ de Samsuiluna informa que, en su noveno año de reinado se produjo la derrota del ejército casita y, en el décimo, la derrota de una coalición liderada por este pueblo.

El texto cuneiforme que aquí se ofrece nos informa de la reconstrucción por parte de Samsuiluna de seis fortalezas, mandadas construir por Sūmû-la-Il y dedicadas a una serie de divinidades que ocupaban un lugar destacado en el panteón religioso babilónico de ese momento. El hecho de que los nombres de las murallas que se citan no aparezcan recogidas en los $<<$ Nombres de año $>>$ de Samsuiluna, no es óbice para constatar la importancia que tanto Samsuiluna como sus predecesores dieron a su política defensiva.

En cuanto a una información más detallada acerca de estas seis murallas y la ubicación exacta de las mismas, habrá que examinar otra documentación escrita de Sūmû-la-Il y Samsuiluna. El texto que aquí se presenta incide en la misma dirección: la importancia que los monarcas mesopotámicos concedían a la política constructora en todas sus variantes. La reconstrucción y construcción de murallas por parte de los diferentes reyes de la Primera Dinastía de Babilonia cumplía una doble función: militar para defenderse ante la presencia continuada del pueblo casita y, en un acto meramente "propagandístico" para honrar a su dios patrono.

\section{Bibliografía}

Borger, R., 1979, Babylonisch-Assyrische Lesestücke, 3 vols. Roma.

Borger, R., 1979, Babylonisch-Assyrische Lesestücke (=AnOr 54), 2 vols. Roma.

Bottéro, J., 2001, La religion más antigua. Mesopotamia, Madrid.

Burkhar, K., 1965, "Igigū und Anunnakkū nach den Akkadischen Quellen”, in H. Güterbock and T. Jacobsen (eds.), Studies in Honor of Benno Landsberger on his Seventy-Fifth Birtday, April 21, 1965. Assyriological Studies, 16, Chicago: Oriental Institute Press.

Brinkman, J.A., 1976-1980, “Kassiten”, RlA 5, pp. 464-473.

De Smet, S.W., 1990, "Kashshû in Old-Babylonian documents", Akkadica 68, pp. 1-19.

Edzard, D. O., 1957, Die 'Zweite Zwischenzeit'Babyloniens, Wiesbaden.

Edzard, D. O., 1965, "Mesopotamien. Die Mythologie der Sumerer und Akkader." in H.W. Haussig (ed.), Götter und Mythen im Vorderen Orient. Wörterbuch der Mythologie, erste Abteilung, Bd. I, Stuttgart, pp. 17-140.

Falkenstein, A., 1965, "Die Anunna in der sumerischen Überlieferung", in H. Güterbock and T. Jacobsen (eds.), Studies in Honor of Benno Landsberger on his Seventy-Fifth Birtday, April 21, 1965. Assyriological Studies, 16, Chicago: Oriental Institute Press.

https://oi.uchicago.edu/sites/oi.uchicago.edu/files/uploads/shared/docs/as16.pdf 
Frayne, D.R., 1990, The Royal Inscriptions of Mesopotamia Early Periods (=RIME) vol.4. Old Babylonian Period (2003-1595 B.C.) University of Toronto Press.

Gibson, McG., 1974, "Violation of fallow and engineered disaster in mesopotamian civilisation", in T. E. Downing and McG. Gibson (eds.), Irrigation's Impact on Society, Tucson, pp. 7-20.

Horsnell, M.J.A., 1999, The Year Names of the First Dynasty of Babylon, 2vols. Hamilton, McMaster University Press.

Jean, Ch. F., 1923, Le milieu biblique avant Jésus-Christ. Vol. 2º, Paris, Geuthner.

Jiménez Zamudio, R., 2002, Antología de textos acadios, Madrid.

Jiménez Zamudio, R., 2015, El Poema de Gilgamesh, Madrid.

Jordi Vidal., 2014, "La Divinización Real en Mesopotamia: Una Teología Política", Arys 12, pp. 31-46.

Kärki, I., 1983, Die sumerischen und akkadischen Königsinschriften der altbabylonischen Zeit II: Babylon (=Studia Orientalia 55/1), Helsinki.

Katz, D., 2003, The Image of the Netherworld in the Sumerian Sources. Bethesda, MD:CDL Press.

King, L.W., 1898-1900, The Letters and Inscriptions of Hammurabi, King of Babylon, about 2000 B.C. to Which Are Added a Series of Letters of Other Kings of the First Dynasty of babylon, 3 vols. (= Luzac's Semitic Text and Translation Series vols.2-3 and 8), London.

Landsberger, B., 1954, “Assyrische Königsliste und Dunkles Zeittalter”, JCS 8, pp. 31-45, 4773 у $106-133$. Madrid

Lara Peinado, F., 1998, Diccionario Biográfico del Mundo Antiguo: Egipto y Próximo Oriente,

López Montero, R., Pino Cano, J. A. y Torres Torres, E., 2014, El prisma de Senaquerib (Chicago OIM A2793): Introducción, Texto Bilingüe y Notas. Madrid.

Márquez Rowe, I., 1997, "Inscripciones reales cuneiformes del II y I milenio a. de C.”, Aula Orientalis 15, pp. 69-78.

Matthews, L.P., 1970, The First Dynasty of Babylon: History and Texts. Birmingham University $\mathrm{PhD}$ Dissertation.

Renger, J.,1979, "Interaction of temple, palace and private enterprise in the Old Babylonian economy" en E. Lipinsky (ed.), State and Temple Economy in the Near East. I, Orientalia Lovaniensia Analecta 5, Leuven, pp.249-503.

Ungnand, A., 1907, Altbabylonische Privaturkunden. Leipzig.

Ungnand, E., 1938, “Datenlisten”. RlA 2 pp. 131-195.

Winckler, H. und Böhden, E., 1892, Altbabylonische Keilschrifttexte zum Gebrauch bei Vorlesungen. Leipzig.

Winckler, H. et al., 1892, Historische Texte altbabylonischer Herrscher. Berlin.

Yoffee, N., 1977, The Economic Role of the Crown in the Old Babylonian Period. Malibu: Bibliotheca Mesopotamica 5). Malibu.

https://janes.scholasticahq.com/article/2317.pdf 


\section{Abreviaturas de revistas e instituciones}

ABK: H. Winckler and E. Böhden, Altabylonische Keilschrifttexte zum Gebrauch bei Vorlesungen (Leipzig 1892).

Akkadica: Akkadica. Périodique bimestriel de la Fondation Assyriologique Georges Dossin (Brussels 1977 ff.); Suppl. = Supplément (Leuven 1983 ff.).

AnOr: Analecta Orientalia (Rome $1931 \mathrm{ff}$.).

ARN: ÇIG, M., KIZILYAY, H., KRAUS, F. R., Eski babil zamanina ait Nippur hukukî vesikalari, Altbabylonische Rechturkunden aus Nippur, Istanbul, 1952.

BAL: R. Borger, Babylonisch-assyrische Lesestücke (= AnOr. 54, ${ }^{1} 1963 ;{ }^{2} 1979$ ).

BE: Babylonian Expedition of the University of Pennsylvania, Series A: Cuneiform Texts, vols.1-14. Philadelphia, 1893-1914.

BM: British Museum. A Guide to the Babylonian and Assyrian Antiquities, 3rd edition. London, 1922.

CT: Cuneiform Texts from Babylonian Tablets in the British Museum. London, 1896-2020.

IM: Museum siglum of the Iraq Museum in Baghdad.

Iraq: A Guide to the Iraq Museum Collections. Baghdad, 1942.

JCS: Journal of Cuneiform Studies. New Haven. Cambridge Mass.

LIH: L. W. King, The Letters and Inscriptions of Hammurabi, King of Babylon, about B.C. 2200, to Which Are Added a Series of Letters of Other Kings of the First Dynasty of Babylon, 1 (London 1898); 2-3 (London 1900).

PBS: University of Pennsylvania, Publications of the Babylonian Section (Philadelphia $1911 \mathrm{ff}$.).

RIA: Reallexikon der Assyriologie. Berlin, 1932-2020.

SAKAZ: K. Oberhuber, Sumerische und akkadische Keilschriftdenkmäler des Archäologischen Museumszu Florenz (= IBK Sonderheft 7/8, 1958/1960).

TCL: Textes cunéiformes du Musée du Louvre, Département des Antiquités Orientales. Paris, 1910-2020.

UET: Ur Excavations, Texts. London, 1928-2020.

VA: Museum siglum of the Vorderasiatisches Museum, Berlin (Vorderasiatische Abteilung, Ass. $=$ Assur $)$.

VAS: Vorderasiatische Schriftdenkmäler der Königlichen Museen zu Berlin (Leipzig 19071917); NF: Vorderasiatische Schriftdenkmäler der Staatlichen Museen zu Berlin (Berlin 1971ff.).

YOS: Yale Oriental Series, Babylonian Texts. New Haven, 1915-2020. 


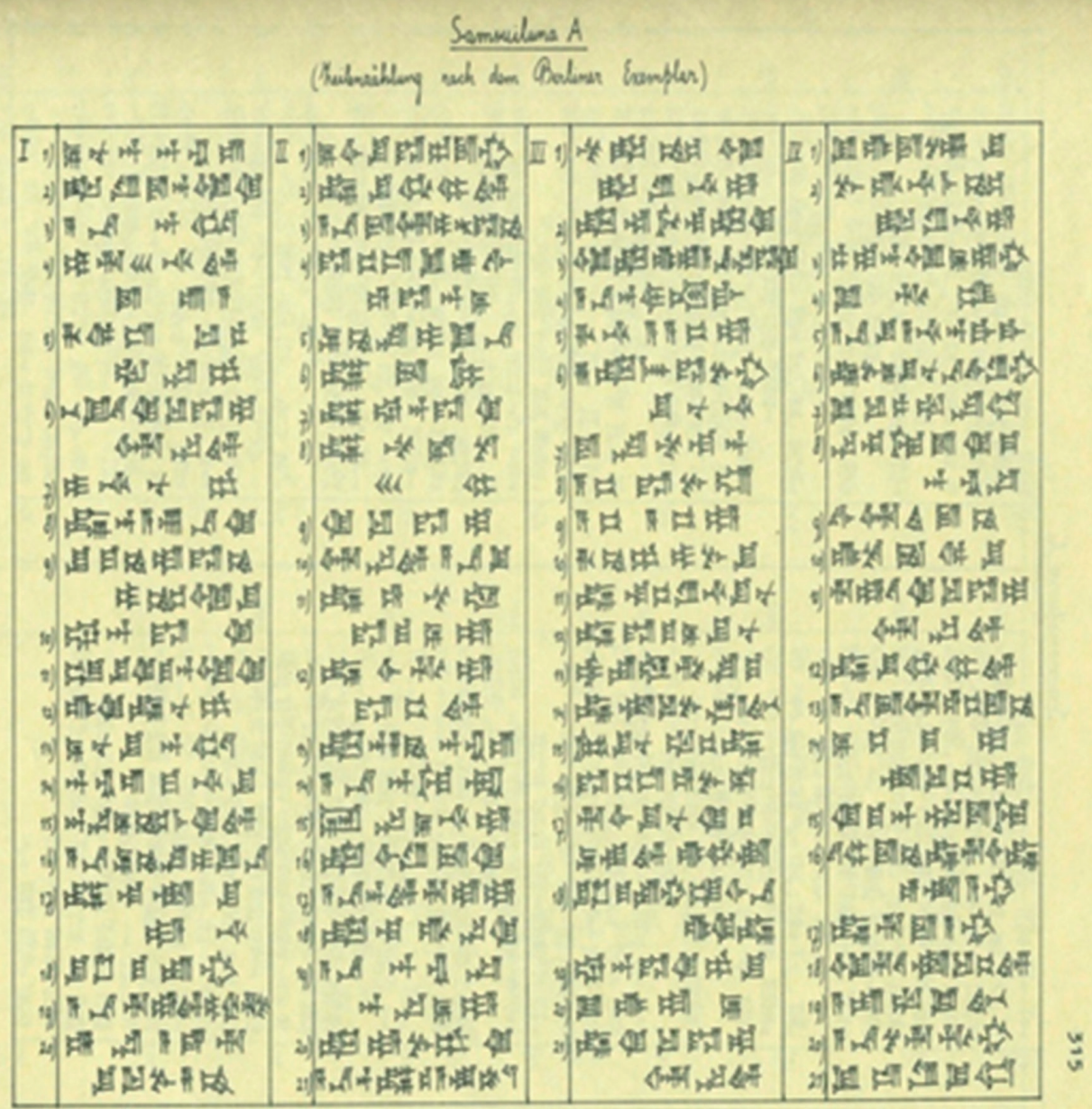

Fig. 1. Plancha cuneiforme de la inscripción real A de Samsuiluna. R. Borger, BabylonischAssyrische Lesestücke. Heft II. Analecta Orientalia 54. Roma, 1979, p. 315. 